\title{
Allergic diseases and long-term risk of autoimmune disorders: longitudinal cohort study and cluster analysis
}

\author{
Mamidipudi Thirumala Krishna $\mathbb{1}^{1,2}$, Anuradhaa Subramanian ${ }^{3}$, \\ Nicola J. Adderley ${ }^{3}$, Dawit T. Zemedikun $\mathbb{1}^{3}$, Georgios V. Gkoutos ${ }^{4,5}$ and \\ Krishnarajah Nirantharakumar ${ }^{3,5}$
}

Affiliations: ${ }^{1}$ Dept of Allergy and Immunology, University Hospitals Birmingham NHS Foundation Trust, Birmingham, UK. ${ }^{2}$ Institute of Immunology and Immunotherapy, University of Birmingham, Birmingham, UK. ${ }^{3}$ Institute of Applied Health Research, University of Birmingham, Birmingham, UK. ${ }^{4}$ Institute of Cancer and Genomics, University of Birmingham, Birmingham, UK. ${ }^{5}$ Midlands Health Data Research UK, Birmingham, UK.

Correspondence: Mamidipudi Thirumala Krishna, Dept of Allergy and Immunology, Birmingham Heartlands Hospital, Bordesley Green East, Birmingham, B9 5SS, UK. E-mail: mtkrishnadyahoo.com

@ERSpublications

This study has shown that the long-term risk of autoimmune disorders is significantly higher in patients with allergic diseases. Allergic diseases and autoimmune disorders co-occur, and show an ageand sex-related clustering pattern. http://bit.ly/33tqkZn

Cite this article as: Krishna MT, Subramanian A, Adderley NJ, et al. Allergic diseases and long-term risk of autoimmune disorders: longitudinal cohort study and cluster analysis. Eur Respir J 2019; 54: 1900476 [https://doi.org/10.1183/13993003.00476-2019].

\section{ABSTRACT}

Introduction: The association between allergic diseases and autoimmune disorders is not well established. Our objective was to determine incidence rates of autoimmune disorders in allergic rhinitis/conjunctivitis (ARC), atopic eczema and asthma, and to investigate for co-occurring patterns.

Methods: This was a retrospective cohort study (1990-2018) employing data extracted from The Health Improvement Network (UK primary care database). The exposure group comprised ARC, atopic eczema and asthma (all ages). For each exposed patient, up to two randomly selected age- and sex-matched controls with no documented allergic disease were used. Adjusted incidence rate ratios (aIRRs) were calculated using Poisson regression. A cross-sectional study was also conducted employing Association Rule Mining (ARM) to investigate disease clusters.

Results: 782320, 1393570 and 1049868 patients with ARC, atopic eczema and asthma, respectively, were included. aIRRs of systemic lupus erythematosus (SLE), Sjögren's syndrome, vitiligo, rheumatoid arthritis, psoriasis, pernicious anaemia, inflammatory bowel disease, coeliac disease and autoimmune thyroiditis were uniformly higher in the three allergic diseases compared with controls. Specifically, aIRRs of SLE (1.45) and Sjögren's syndrome (1.88) were higher in ARC; aIRRs of SLE (1.44), Sjögren's syndrome (1.61) and myasthenia (1.56) were higher in asthma; and aIRRs of SLE (1.86), Sjögren's syndrome (1.48), vitiligo (1.54) and psoriasis (2.41) were higher in atopic eczema. There was no significant effect of the three allergic diseases on multiple sclerosis or of ARC and atopic eczema on myasthenia. Using ARM, allergic diseases clustered with multiple autoimmune disorders. Three age- and sex-related clusters were identified, with a relatively complex pattern in females $\geqslant 55$ years old.

Conclusions: The long-term risks of autoimmune disorders are significantly higher in patients with allergic diseases. Allergic diseases and autoimmune disorders show age- and sex-related clustering patterns. 


\section{Introduction}

Autoimmune disorders and allergic diseases are characterised by an immune dysregulated state $[1,2]$. From an immunological viewpoint, it has been suggested that these conditions are largely polarised with disparate mechanisms, with autoimmune disorders and allergic diseases primarily mediated by a T-helper (Th) 1 and Th2 cellular immune response, respectively $[3,4]$. Both have a complex aetio-pathogenesis and occur as a consequence of a complex interplay between multiple genetic and environmental factors with other possible variables hitherto unknown [5].

Western countries have been challenged with an "allergy epidemic" during the last three to four decades, resulting in a very high burden of allergic rhinitis, allergic conjunctivitis, atopic eczema and asthma $[6,7]$. In particular, the UK ranks as one of the highest countries with respect to the prevalence of allergic diseases worldwide [7]. Interestingly, there has been a parallel increase in the incidence rates of several autoimmune disorders, including multiple sclerosis, inflammatory bowel disease, rheumatoid arthritis and systemic lupus erythematosus (SLE), during the same period [5]. The biological plausibility and causality hypothesis has linked these observations to the possible involvement of common triggers such as infection, genetic foci, environmental factors, reduced biodiversity and birth by caesarean section [8]. Furthermore, there is some emerging evidence regarding an enhanced risk of autoimmune disorders such as SLE, rheumatoid arthritis, Sjögren's syndrome, multiple sclerosis, coeliac disease and myasthenia gravis in patients with allergic diseases and vice versa [9-12]. Studies carried out in South Asian immigrants in Western countries have shown higher rates of both allergic diseases and autoimmune disorders, with a positive association with the duration of settlement away from their native country and with an enhanced risk in their offspring [13-15]. These studies have, however, been limited by relatively small sample size, methodological issues, selection bias, focus on a single or limited number of allergic diseases and/or autoimmune disorders, and some by not accounting for important confounders such as smoking history. Nonetheless, the evidence generated from these studies has challenged the Th1/Th2 paradigm $[4,16]$.

The main aim of this study was to test the hypothesis that an underlying allergic disease such as allergic rhinitis/conjunctivitis (ARC), atopic eczema or asthma enhances the risk of development of an autoimmune disorder. In a large population-based study, we determined adjusted incidence rate ratios (aIRRs) of common autoimmune disorders in patients with and without ARC, atopic eczema and asthma, and investigated for clustering patterns between allergic diseases and autoimmune disorders.

\section{Methods}

\section{Setting}

Data were extracted from The Health Improvement Network (THIN), a database comprised of electronic medical records from UK general practices using Vision software (www.visionhealth.co.uk). It includes coded data recorded by the patient's general practitioner for clinical and management purposes, capturing every consultation. Symptoms and diagnoses are recorded using Read Codes, a hierarchical coding system for structured storage information [17]. 15 million patients at 787 general practices across the UK have contributed data to THIN; there are approximately 3 million active patients comprising $6-7 \%$ of the population. The data extracted from THIN are generalisable to the UK population for major health conditions [18].

In order to ensure data quality and allow sufficient time to document important covariates, general practices were included in this study only if they met the following criteria: 1) 12 months after the practice reported an acceptable mortality rate [19] and 2) 12 months after date the practice began using electronic medical records, and study start date.

\section{Ethics approval}

The THIN data collection scheme and research carried out using THIN data were approved by the National Health Service (NHS) South-East Multicentre Research Ethics Committee in 2003. Under the terms of the approval, studies must undergo independent scientific review. Approval for this study was obtained from the Scientific Review Committee (for the use of THIN data) in August 2018 (SRC reference 18THIN64).

\section{Study design}

Incidence of autoimmune disorders

A longitudinal retrospective cohort study was carried out (January 1, 1990-January 17, 2018). Patients of all ages registered for at least 365 days prior to study entry were included.

The exposed cohort was defined as patients with a prevalent or incident diagnosis of any one or more of the three allergic diseases of interest: asthma, ARC and atopic eczema. Food allergy was not included in our analysis, as the diagnostic coding may not be accurate in primary care records, unless patients had undergone a review by an allergy specialist. In a sensitivity analysis, the exposed cohort was defined as 
incident diagnoses only (newly diagnosed after registration with the practice). For patients with multiple allergic diseases, the earliest diagnosis was considered as the index condition. Diagnosis of all allergic diseases was indicated by the presence of a corresponding clinical (Read) code in the patient's medical record. Allergic rhinitis and allergic conjunctivitis were treated as a single exposure group (i.e. ARC) due to high rates of co-occurrence and as their inflammatory responses in the mucosa and conjunctiva unfold similarly [20, 21].

For each exposed patient, up to two controls were randomly selected within the same general practice from an age- (within 1 year) and sex-matched pool of patients with no allergic disease diagnosis. The following method was used to randomly select matched controls. Patients with an allergic disease were identified and their order was shuffled by randomly permuting the patient list (following the Fisher-Yates algorithm), using a linear congruential generator as the source of randomness. All permutations occur with equal likelihood. Following this, controls were selected. Shuffling ensures that all patients in the exposure group have an equal chance of being matched to a control in instances where that control could potentially be matched with more than one exposed patient. When the number of possible controls for a particular exposed patient exceeded the number required (i.e. $\mathrm{n}=2$ ), a linear congruential generator was used to generate a random number between 1 and the number of potential controls; the potential control at the position of this random number was selected and the process was repeated for subsequent controls.

Outcomes were the following autoimmune disorders (considered separately): SLE, Sjögren's syndrome, vitiligo, rheumatoid arthritis, psoriasis, pernicious anaemia, myasthenia gravis, inflammatory bowel disease, coeliac disease, autoimmune thyroiditis (AIT) and multiple sclerosis.

The index date for exposed patients was the date of diagnosis of the first allergic disease recorded for newly diagnosed patients or date of study entry for patients with an existing diagnosis. Unexposed controls were assigned the same index date as their corresponding exposed patient to avoid immortal time bias [22]. Exposed and control patients were followed from the index date until the earliest of the following end-points: date of outcome recording (autoimmune disorder of interest), date of death, date patient left the practice, date the practice ceased contributing to THIN or study end date.

\section{Read Codes}

Read Code lists (see supplementary material) for each condition were compiled as follows: relevant codes were extracted from a database containing all current and past version 2 Read Codes; each code was reviewed and assessed for relevance; where available, code lists in previously published literature [23-26] were used to inform and validate code selection; in the case of uncertainty regarding the relevance of a code, consensus was reached in consultation with an experienced primary care physician.

\section{Disease clusters}

A cross-sectional study was performed with a census date of January 1, 2018. Patients of all ages who had been registered with the practice for at least 365 days were included.

\section{Definitions of covariates}

Age, sex, body mass index (BMI), deprivation quintile, ethnicity and smoking status were included as covariates in the cohort analyses. For BMI and smoking status, the last value recorded prior to the index date was used. BMI was categorised as $<18.5 \mathrm{~kg} \cdot \mathrm{m}^{-2}$ (underweight), $18-25 \mathrm{~kg} \cdot \mathrm{m}^{-2}$ (normal weight), $25-30 \mathrm{~kg} \cdot \mathrm{m}^{-2}$ (overweight) and $>30 \mathrm{~kg} \cdot \mathrm{m}^{-2}$ (obese). Smoking status was categorised as nonsmoker, ex-smoker and current smoker. Social deprivation was recorded as Townsend deprivation quintile [27]. Missing BMI, deprivation quintile, ethnicity and smoking data were handled by employing a "missing" category within the corresponding categorical variables.

\section{Analysis \\ Incidence of autoimmune disorders}

Baseline covariates were summarised for patients in the three separate exposure groups (asthma, atopic eczema and ARC) and their corresponding controls using appropriate descriptive statistics: mean with standard deviation and median (interquartile range (IQR)) for continuous variables, and number (percentage) for categorical variables. Incidence rates of each autoimmune disorder in each of the three allergic diseases were calculated by dividing the number of patients with a newly diagnosed autoimmune disorder (numerator) by the total number of person-years at risk (denominator) for the given allergic disease. Crude IRRs and aIRRs together with their corresponding 95\% confidence intervals were calculated using Poisson regression. Separate Poisson regression models were used for each allergic disease/ autoimmune disorder combination. All models were adjusted for the following covariates: age, sex, BMI, ethnicity, Townsend deprivation quintile and smoking status. Two-sided p-values were calculated; $\mathrm{p}<0.05$ 
was considered to indicate statistical significance. All analyses were performed in Stata/IC version 14 (StataCorp, College Station, TX, USA).

The study flow diagram is shown in figure 1 .

\section{Disease clusters}

Association Rule Mining (ARM) was used to assess how the three allergic diseases associated with the 11 autoimmune disorders, as described by ZEMEDikun et al. [28]. This is a data mining technique used to investigate frequent co-occurring associations among variables in large databases. Association rules were created specifying allergic diseases as antecedent and autoimmune disorders as consequent so that the reverse associations were not detected. Significance was measured using three parameters: "support" (how frequently the disease combination appears in the dataset), "confidence" (the conditional probability that a subject with the antecedent disease will also have the consequent condition) and "lift" (the ratio of the observed support to that expected if the two events were independent). The minimum parameter thresholds for "support" and "confidence" were set at 0.0005 to extract sufficient number of association rules. "Lift", also referred to as the interestingness measure, measures the importance of a relationship. Specifically, a "lift" of $>1.0$ indicates that the antecedent and consequent condition appear more often than expected, i.e. the antecedent (allergic disease) exerts a positive effect on the occurrence of the consequent (autoimmune disorder). ARM analysis was performed on the full cross-sectional dataset, and separately for age- and sex-restricted subgroups. ARM analysis was performed using R version 3.3.1 (www.r-project.org).

\section{Results}

\section{Incidence of autoimmune disorders}

782320,1393570 and 1049868 patients with ARC, atopic eczema and asthma, respectively, together with their matched controls, were included in the analyses (figure 1). Mean \pm SD age at baseline was $36.4 \pm 19.4$, $29.8 \pm 25.3$ and $35.6 \pm 21.3$ years in patients with ARC, atopic eczema and asthma, respectively; $47.1 \%, 45.9 \%$ and $47.9 \%$ were male, respectively. Baseline sex, smoking status, BMI, ethnicity and Townsend deprivation quintile were similar between patients with each allergic disease and their respective controls, whereas

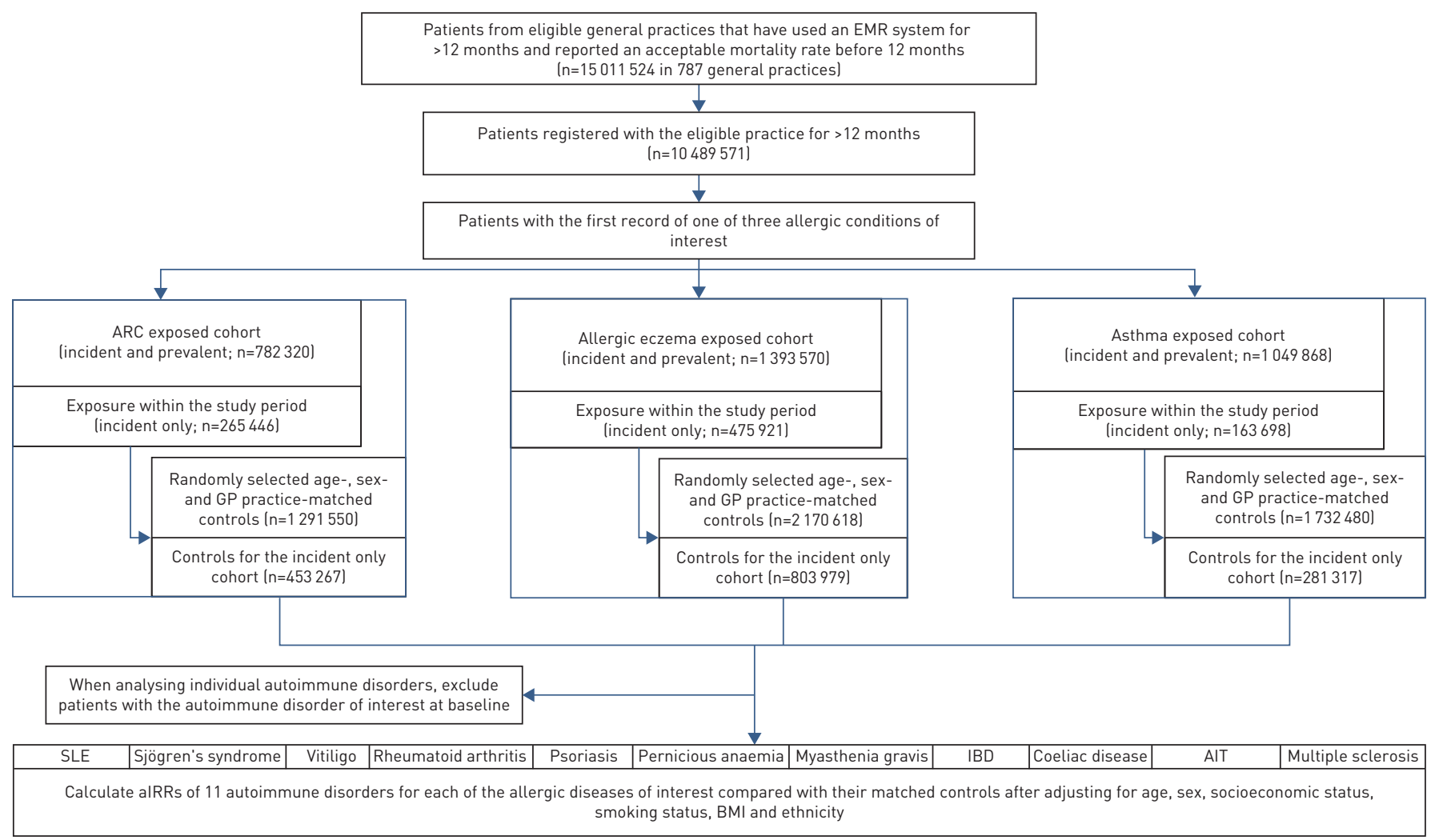

FIGURE 1 Flow diagram summarising study design. EMR: electronic medical record; ARC: allergic rhinitis/conjunctivitis; GP: general practitioner; SLE: systemic lupus erythematosus; IBD: inflammatory bowel disease; AIT: autoimmune thyroiditis; alRR: adjusted incidence rate ratio; BMI: body mass index. 
unexposed controls were slightly older than their corresponding exposure group (table 1); this arose as a result of incomplete matching of some patients due to lack of availability of matched controls within the same practice. Patients with allergic diseases had more autoimmune disorders at baseline.

TABLE 1 Baseline patient characteristics

\begin{tabular}{|c|c|c|c|c|c|c|}
\hline & \multicolumn{2}{|c|}{ ARC } & \multicolumn{2}{|c|}{ Atopic eczema } & \multicolumn{2}{|c|}{ Asthma } \\
\hline Subjects & 782320 & 1291550 & 1393570 & 2170618 & 1049868 & 1732480 \\
\hline Age years & $36.44 \pm 19.44$ & $38.14 \pm 19.11$ & $29.77 \pm 25.30$ & $32.74 \pm 25.45$ & $35.61 \pm 21.26$ & $37.98 \pm 20.91$ \\
\hline \multicolumn{7}{|l|}{ Age categories } \\
\hline $0-9$ years & 56995 (7.29) & 78503 (6.08) & $443272(31.81)$ & $602343(27.75)$ & $110635(10.54)$ & $142078(8.20)$ \\
\hline $30-39$ years & $165941(21.21)$ & 292551 (22.65) & 157265 (11.29) & $272643(12.56)$ & $178980(17.05)$ & 323138 (18.65) \\
\hline 40-49 years & $115694(14.79)$ & $209110(16.19)$ & $126174(9.05)$ & $226457(10.43)$ & 126552 (12.05) & $234123(13.51)$ \\
\hline $50-59$ years & $78520(10.04)$ & 141956 (10.99) & $109781(7.88)$ & $198495(9.14)$ & 98207 (9.35) & $182006(10.51)$ \\
\hline $60-69$ years & $58414(7.47)$ & $103498(8.01)$ & $96955(6.96)$ & $171496(7.90)$ & $81459(7.76)$ & $147338(8.50)$ \\
\hline$\geqslant 70$ years & $52619(6.73)$ & $93002(7.20)$ & $130935(9.40)$ & $230393(10.61)$ & $89569(8.53)$ & $163033(9.41)$ \\
\hline \multicolumn{7}{|l|}{ Sex } \\
\hline Underweight (<18.5 $\mathrm{kg} \cdot \mathrm{m}^{-2}$ ) & $13778(1.76)$ & 24658 (1.91) & $18525(1.33)$ & $32012(1.47)$ & $18722(1.78)$ & $32606(1.88)$ \\
\hline Normal weight $\left(18.5-25 \mathrm{~kg} \cdot \mathrm{m}^{-2}\right)$ & $240550(30.75)$ & 391963 (30.35) & 296729 (21.29) & $485257(22.36)$ & $273231(26.03)$ & $488611(28.20)$ \\
\hline Overweight $\left(25-30 \mathrm{~kg} \cdot \mathrm{m}^{-2}\right)$ & $167639(21.43)$ & 260249 (20.15) & $215020(15.43)$ & $345277(15.91)$ & $205041(19.53)$ & $323882(18.69)$ \\
\hline Obese $\left(>30 \mathrm{~kg} \cdot \mathrm{m}^{-2}\right)$ & $95186(12.17)$ & $148894(11.53)$ & $131161(9.41)$ & $200892(9.26)$ & 156489 (14.91) & $189050(10.91)$ \\
\hline Missing & $265167(33.89)$ & $465786(36.06)$ & $732135(52.54)$ & $1107180(51.01)$ & $396385(37.76)$ & $698331(40.31)$ \\
\hline \multicolumn{7}{|l|}{ Smoker categories } \\
\hline Nonsmoker & $393523(50.30)$ & 581795 (45.05) & 472948 (33.94) & $762193(35.11)$ & $454001(43.24)$ & $735619(42.46)$ \\
\hline Ex-smoker & $103476(13.23)$ & $158144(12.24)$ & $151830(10.90)$ & $228211(10.51)$ & $153849(14.65)$ & $198447(11.45)$ \\
\hline Current smoker & $117214(14.98)$ & 255481 (19.78) & $181665(13.04)$ & $304165(14.01)$ & $202006(19.24)$ & 329486 (19.02) \\
\hline Missing & $168107(21.49)$ & $296130(22.93)$ & $587127(42.13)$ & $876049(40.36)$ & $240012(22.86)$ & $468928(27.07)$ \\
\hline \multicolumn{7}{|l|}{ Ethnicity } \\
\hline Caucasian & $276634(35.36)$ & 419125 (32.45) & 501207 (35.97) & $737165(33.96)$ & 398059 (37.92) & 543068 (31.35) \\
\hline 5 (most deprived) & $87912(11.24)$ & $154989(12.00)$ & $160308(11.50)$ & $256742(11.83)$ & $189185(13.33)$ & $212347(12.26)$ \\
\hline Missing & $112743(14.41)$ & $184220(14.26)$ & $200841(14.41)$ & $314144(14.47)$ & $159252(15.17)$ & $257959(14.89)$ \\
\hline \multicolumn{7}{|c|}{ Autoimmune disorders at baseline } \\
\hline Systemic lupus erythematosus & $791(0.10)$ & 1195 (0.09) & $1445(0.10)$ & $1610(0.07)$ & $1064(0.10)$ & $1514(0.09)$ \\
\hline Sjögren's syndrome & $516(0.07)$ & $511(0.04)$ & $693(0.05)$ & $813(0.04)$ & $572(0.05)$ & $735(0.04)$ \\
\hline Vitiligo & $2191(0.28)$ & $2516(0.19)$ & $3532(0.25)$ & $3519(0.16)$ & $1996(0.19)$ & $2895(0.17)$ \\
\hline Rheumatoid arthritis & $3819(0.49)$ & $6122(0.47)$ & $6933(0.50)$ & $10321(0.48)$ & $6310(0.60)$ & $8576(0.50)$ \\
\hline Psoriasis & $20077(2.57)$ & $28648(2.22)$ & 40319 (2.89) & $39147(1.80)$ & $24362(2.32)$ & $35489(2.05)$ \\
\hline Pernicious anaemia & $1458(0.19)$ & $2095(0.16)$ & $2879(0.21)$ & $4101(0.19)$ & $2464(0.23)$ & $3166(0.18)$ \\
\hline Myasthenia gravis & $180(0.02)$ & $256(0.02)$ & $251(0.02)$ & $460(0.02)$ & $273(0.03)$ & $349(0.02)$ \\
\hline Inflammatory bowel disease & $5003(0.64)$ & $7030(0.54)$ & $7848(0.56)$ & $9537(0.44)$ & $6428(0.61)$ & $8843(0.51)$ \\
\hline Coeliac disease & $1824(0.23)$ & $2141(0.17)$ & $2876(0.21)$ & $3221(0.15)$ & 2398 (0.23) & $2770(0.16)$ \\
\hline Autoimmune thyroiditis & $2069(0.26)$ & $2586(0.20)$ & $2528(0.18)$ & $3456(0.16)$ & $2291(0.22)$ & $3215(0.19)$ \\
\hline Multiple sclerosis & $1479(0.19)$ & $2474(0.19)$ & $2204(0.16)$ & $3439(0.16)$ & $1519(0.14)$ & $3147(0.18)$ \\
\hline
\end{tabular}

Data are presented as $\mathrm{n}$, mean \pm SD or $\mathrm{n}(\%)$. ARC: allergic rhinitis/conjunctivitis; BMI: body mass index. 
In patients with ARC compared with patients with no diagnosed allergic disease, aIRRs were: SLE 1.45 (95\% CI 1.27-1.67), Sjögren's syndrome 1.88 (95\% CI 1.63-2.17), vitiligo 1.36 (95\% CI 1.25-1.47), rheumatoid arthritis 1.21 (95\% CI 1.15-1.28), psoriasis 1.38 (95\% CI $1.34-1.42$ ), pernicious anaemia 1.30 (95\% CI 1.20-1.40), inflammatory bowel disease 1.33 (95\% CI 1.26-1.41), coeliac disease 1.39 (95\% CI 1.28-1.51) and AIT 1.18 (95\% CI 1.08-1.29) (figure 2 and supplementary table E1).

In patients with atopic eczema, aIRRs were: SLE 1.86 (95\% CI 1.66-2.09), Sjögren's syndrome 1.48 (95\% CI 1.30-1.69), vitiligo 1.54 (95\% CI 1.44-1.64), rheumatoid arthritis 1.28 (95\% CI 1.22-1.34), psoriasis 2.41 (2.36-2.46), pernicious anaemia 1.25 (95\% CI 1.18-1.33), inflammatory bowel disease 1.53 (95\% CI 1.45-1.61), coeliac disease 1.41 (95\% CI 1.32-1.50) and AIT 1.13 (95\% CI 1.05-1.22) (figure 2 and supplementary table E1).

In patients with asthma, aIRRs were: SLE 1.44 (95\% CI 1.27-1.62), Sjögren's syndrome 1.61 (95\% CI 1.411.84), vitiligo 1.24 (95\% CI 1.15-1.34), rheumatoid arthritis 1.44 (95\% CI 1.37-1.50), psoriasis 1.39 (95\% CI 1.36-1.43), pernicious anaemia 1.30 (95\% CI 1.22-1.38), myasthenia gravis 1.56 (95\% CI 1.29-1.88), inflammatory bowel disease 1.44 (95\% CI 1.37-1.51), coeliac disease 1.44 (95\% CI 1.34-1.55) and AIT 1.23 (95\% CI 1.14-1.33) (figure 2 and supplementary table E1).

There was no statistically significant difference in incidence of multiple sclerosis in any of the allergic diseases or of myasthenia gravis in ARC and atopic eczema.

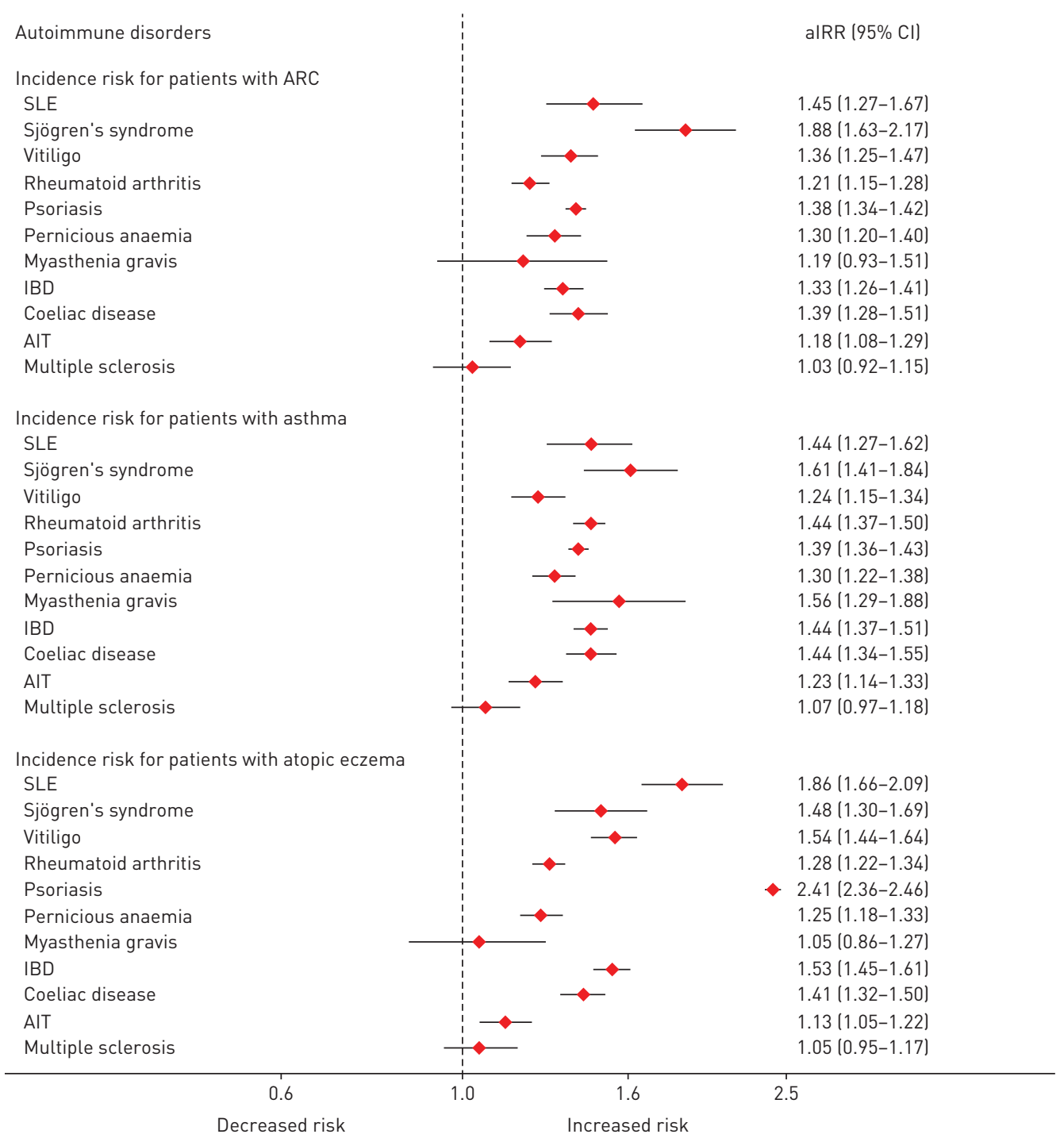

FIGURE 2 Forest plots summarising adjusted incidence rate ratios (alRRs) of autoimmune disorders in each allergic disease (incident and prevalent cases). ARC: allergic rhinitis/conjunctivitis; SLE: systemic lupus erythematosus; IBD: inflammatory bowel disease; AIT: autoimmune thyroiditis. 
Supplementary figure E1 summarises adjusted hazard ratios of autoimmune disorders in combinations of one, two and three allergic diseases. A sensitivity analysis including incident diagnoses of allergic diseases made only little difference to the results (supplementary table E2).

\section{Disease clusters}

In the ARM analysis of all ages and both sexes, all three allergic diseases clustered with psoriasis, coeliac disease and inflammatory bowel disease. ARC and atopic eczema clustered with vitiligo; ARC and asthma clustered with rheumatoid arthritis and AIT; and asthma and atopic eczema clustered with pernicious anaemia. The associations between ARC and vitiligo and between atopic eczema and psoriasis were particularly notable, with lifts of 1.583 and 1.414, respectively, indicating that the probability of vitiligo is $58 \%$ greater in the presence of ARC and the probability of psoriasis is $41 \%$ greater in the presence of atopic eczema. The latter association was strengthened when ARC, asthma or both were present (figure 3 and table 2).

Subgroup analysis highlighted three age- and sex-related clusters, $<30,30-54$ and $\geqslant 55$ years, with a relatively complex pattern of association in females $\geqslant 55$ years (supplementary tables E3-E8, and supplementary figures E2 and E3). The three allergic diseases clustered with vitiligo, coeliac disease, psoriasis, pernicious anaemia, inflammatory bowel disease, AIT and rheumatoid arthritis. In males, the

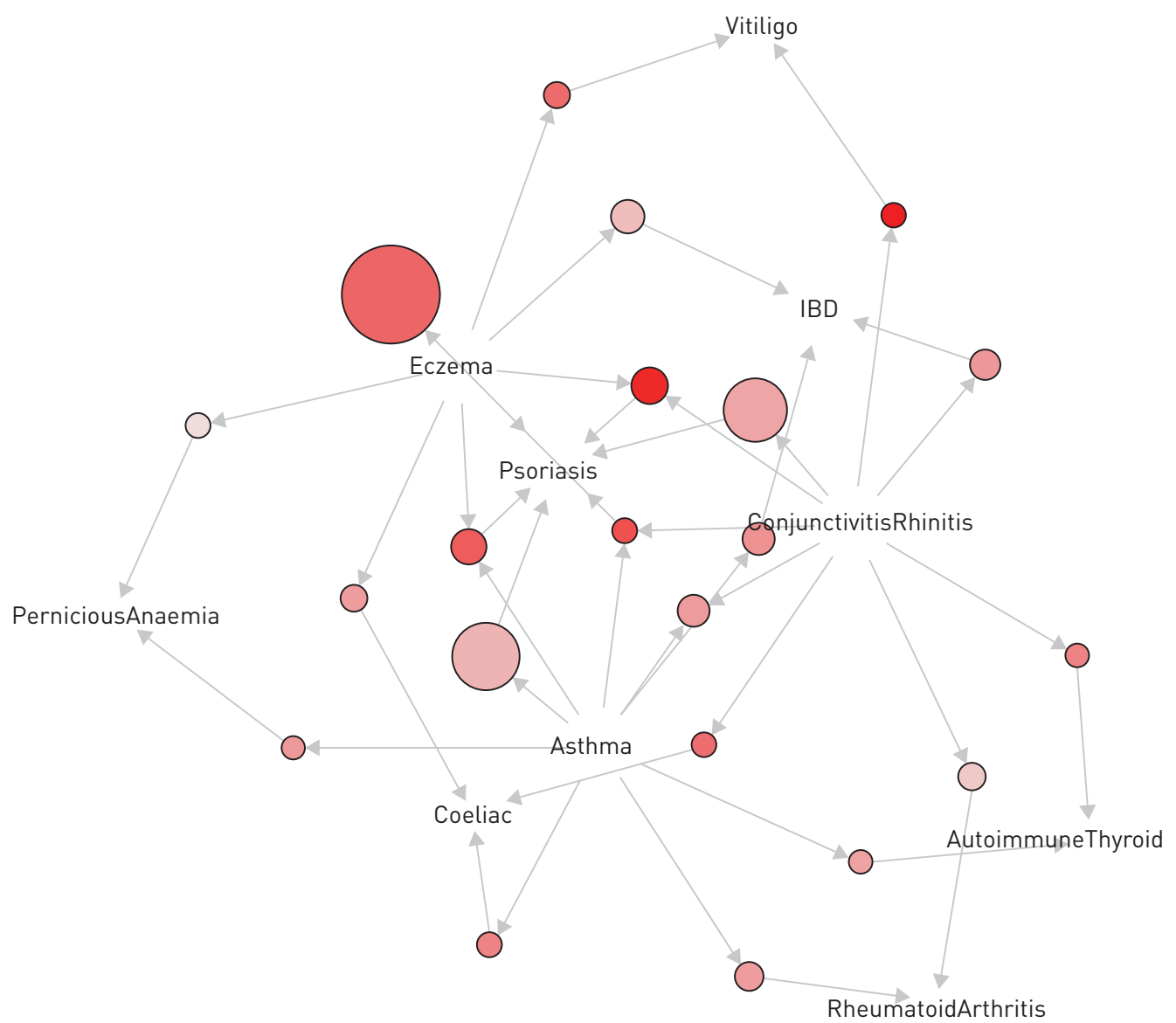

FIGURE 3 Association Rule Mining analysis of the entire study population illustrating clustering and association between allergic diseases and autoimmune disorders. IBD: inflammatory bowel disease. ConjunctivitisRhinitis corresponds to allergic rhinitis/conjunctivitis (ARC). This figure should be reviewed in conjunction with table 2. Each arrow connects an allergic disease (antecedent) with an autoimmune disorder (consequent) via nodes. The size and the intensity of colour within each node represent the level of "support" and level of "lift", respectively (i.e. the larger the node and darker the colour, the greater the "support" and "lift", respectively). For example, there is clustering between asthma, atopic eczema and ARC and psoriasis; asthma and ARC (but not atopic eczema) with rheumatoid arthritis; atopic eczema and ARC (but not asthma) with vitiligo. This suggests that these allergic diseases have a positive effect on the occurrence of the respective autoimmune disorders. 
TABLE 2 Cluster analysis: association rules of allergic diseases and autoimmune disorders in the full cross-sectional dataset (in order of "lift")

\begin{tabular}{|c|c|c|c|c|c|c|}
\hline Rule & Antecedent & & Consequent & Support & Confidence & Lift \\
\hline 1 & \{ConjunctivitisRhinitis\} & $\rightarrow$ & $\{$ Vitiligo $\}$ & 0.0006 & 0.0050 & 1.5835 \\
\hline 2 & \{Eczema,ConjunctivitisRhinitis\} & $\rightarrow$ & \{Psoriasis\} & 0.0020 & 0.0555 & 1.5547 \\
\hline 3 & $\{$ Asthma,Eczema,ConjunctivitisRhinitis\} & $\rightarrow$ & $\{$ Psoriasis\} & 0.0007 & 0.0524 & 1.4654 \\
\hline 4 & $\{$ Asthma,Eczema\} & $\rightarrow$ & $\{$ Psoriasis\} & 0.0018 & 0.0512 & 1.4340 \\
\hline 5 & $\{$ Eczema $\}$ & $\rightarrow$ & \{Psoriasis\} & 0.0090 & 0.0505 & 1.4141 \\
\hline 6 & $\{$ Eczema\} & $\rightarrow$ & $\{$ Vitiligo $\}$ & 0.0008 & 0.0044 & 1.4019 \\
\hline 7 & \{ConjunctivitisRhinitis\} & $\rightarrow$ & $\{$ Coeliac\} & 0.0006 & 0.0054 & 1.3910 \\
\hline 8 & \{ConjunctivitisRhinitis\} & $\rightarrow$ & \{AutoimmuneThyroid\} & 0.0006 & 0.0048 & 1.3427 \\
\hline 9 & $\{$ Asthma $\}$ & $\rightarrow$ & $\{$ Coeliac $\}$ & 0.0007 & 0.0052 & 1.3386 \\
\hline 10 & \{Asthma\} & $\rightarrow$ & $\{\mid B D\}$ & 0.0014 & 0.0110 & 1.3114 \\
\hline 11 & \{ConjunctivitisRhinitis\} & $\rightarrow$ & $\{\mid \mathrm{BD}\}$ & 0.0013 & 0.0109 & 1.2968 \\
\hline 12 & $\{$ Asthma\} & $\rightarrow$ & $\{$ PerniciousAnaemia $\}$ & 0.0006 & 0.0044 & 1.2939 \\
\hline 13 & \{Asthma,ConjunctivitisRhinitis\} & $\rightarrow$ & \{Psoriasis\} & 0.0015 & 0.0459 & 1.2857 \\
\hline 14 & $\{$ Eczema $\}$ & $\rightarrow$ & $\{$ Coeliac $\}$ & 0.0009 & 0.0049 & 1.2785 \\
\hline 15 & $\{$ Asthma\} & $\rightarrow$ & \{RheumatoidArthritis\} & 0.0012 & 0.0091 & 1.2768 \\
\hline 16 & $\{$ Asthma $\}$ & $\rightarrow$ & \{AutoimmuneThyroid\} & 0.0006 & 0.0045 & 1.2527 \\
\hline 17 & \{ConjunctivitisRhinitis\} & $\rightarrow$ & $\{$ Psoriasis\} & 0.0052 & 0.0443 & 1.2403 \\
\hline 18 & \{Asthma & $\rightarrow$ & \{Psoriasis\} & 0.0054 & 0.0423 & 1.1834 \\
\hline 19 & $\{$ Eczema\} & $\rightarrow$ & $\{\mid B D\}$ & 0.0017 & 0.0095 & 1.1381 \\
\hline 20 & \{ConjunctivitisRhinitis\} & $\rightarrow$ & \{RheumatoidArthritis\} & 0.0009 & 0.0078 & 1.0945 \\
\hline 21 & $\{$ Eczema\} & $\rightarrow$ & $\{$ PerniciousAnaemia $\}$ & 0.0006 & 0.0035 & 1.0136 \\
\hline
\end{tabular}

IBD: inflammatory bowel disease. ConjunctivitisRhinitis corresponds to allergic rhinitis/conjunctivitis.

strongest associations were between ARC and vitiligo ( $<30$ years) and between atopic eczema and psoriasis ( $>30$ years). In females, the strongest associations were between asthma/other allergic diseases and psoriasis or inflammatory bowel disease.

\section{Discussion}

\section{Strengths}

To the best of our knowledge, this is the largest study conducted thus far to systematically investigate associations between each allergic disease independently with a range of common autoimmune disorders and to report multimorbidity and clustering patterns. Our data cover a wide geographical area of the UK and are generalisable to the British population [18]. Importantly, our study was conducted in a primary care setting, thereby reducing selection bias. Patients with missing data for some covariates were included in order to maximise the study population and ensure generalisability.

\section{Limitations}

First, the study involved retrospective data extraction. Second, multiple clinicians have contributed to the THIN database and differences in clinical practice, and changes in governance systems, service framework and clinical practice standards over three decades, are possible confounders. Third, there may be lack of standardisation with respect to diagnosis and management criteria in such a large population study involving a wide geographical area. Fourth, the majority of disorders investigated are heterogeneous in nature and it was not within the scope of this study to characterise them. Fifth, atopic status and autoimmune serology for autoimmune disorders could not be captured from the THIN database. Specifically, our data cannot differentiate between allergic asthma and nonallergic asthma in the asthma cohort. Sixth, this study did not specifically investigate the effect of parental allergy.

\section{Interpretation of data}

This study showed higher aIRRs of common autoimmune disorders in patients with ARC, asthma and atopic eczema compared with the control (unexposed) population. Furthermore, ARM analysis applied on the entire study population showed significant clustering between ARC, asthma and atopic eczema and autoimmune disorders including vitiligo, coeliac disease, psoriasis, pernicious anaemia, inflammatory bowel disease, AIT and rheumatoid arthritis. Further subgroup analysis highlighted three age- and sex-related clusters, i.e. $<30,30-54$ and $\geqslant 55$ years, with a relatively more complex pattern of association in older patients, females in particular. The clustering patterns could at least in part be influenced by known 
demographic patterns involving autoimmune disorders, but it is plausible that patients with coexisting allergic diseases and autoimmune disorders represent a distinct phenotype with a specific genetic determinant(s).

While the aIRRs of both organ-specific and systemic autoimmune disorders were uniformly higher in patients with all allergic diseases, effect sizes were greatest in the atopic eczema cohort, with the effect most pronounced for psoriasis, SLE, Sjögren's syndrome, inflammatory bowel disease and vitiligo. Similarly, ARC was positively associated with all autoimmune disorders under consideration, but aIRRs were greatest for SLE and Sjögren's syndrome. A similar pattern was also noted in the asthma cohort, with aIRRs greatest for Sjögren's syndrome, rheumatoid arthritis, myasthenia gravis, coeliac disease and inflammatory bowel disease. The effects on respective autoimmune disorders were more pronounced in the presence of allergic comorbidity (i.e. two or more allergic diseases). Interestingly, aIRRs for multiple sclerosis were uniformly low across all allergic diseases and this is in keeping with a recent study by FAKIH et al. [29].

\section{Comparison with previous studies}

Strong associations with multiple autoimmune disorders and asthma have been reported in patients with coeliac disease [30]. Studies carried out using the National Health Insurance Research database in Taiwan reported a higher incidence of asthma in patients with Sjögren's syndrome [9]. Similar associations were also described between the three allergic diseases and SLE, and our findings are in keeping with these observations [10]. Another study from Taiwan showed a strong association between allergic conjunctivitis and myasthenia gravis [12]. A further study involving 155311 patients with allergic rhinitis, atopic eczema and asthma (considered in a single group) showed positive associations with SLE, rheumatoid arthritis, Sjögren's syndrome, dermatomyositis/polymyositis and systemic sclerosis, with a particularly strong association with Sjögren's syndrome [11].

\section{Biological plausibility}

A number of common factors have been implicated in the aetio-pathogenesis of allergic diseases and autoimmune disorders, including reduced biodiversity, particularly of the gut microbiome, urbanisation, lifestyle factors, diet (including vitamin D status), and recently identified novel metabolic and immune dysregulatory pathways involving mitochondrial stress and intracellular and extracellular RNAs [8]. A meta-analysis of two genome-wide association studies [31] involving allergen sensitisation and self-reported allergy combined with publicly available genome-wide association study data on autoimmune disorders reported commonalities with respect to susceptibility foci, genetic pathways and genomic regulatory sites. Candidate genes have been reported between allergic diseases and autoimmune disorders [32-34]. There is some evidence for an "autoreactive" immunological state in atopic eczema [35], and for involvement of Th17 cells in allergic diseases and autoimmune disorders [36, 37].

\section{Conclusions}

The risk of autoimmune disorder(s) is significantly higher in patients with an underlying allergic disease. Allergic diseases and autoimmune disorders co-occur with an age- and sex-related clustering pattern. Further studies in well-characterised patients involving genomics are needed to confirm these observations and unravel novel mechanisms to pave the way for novel biomarkers, precision and personalised medicine, and primary and secondary prevention strategies in allergic diseases and autoimmune disorders.

Acknowledgements: The authors thank A.P. Huissoon and R. Baretto (University Hospitals Birmingham NHS Foundation Trust, Birmingham, UK) for their comments and feedback on the draft manuscript.

Conflict of interest: M.T. Krishna reports support from Thermo Fisher (sponsorship to attend EAACI conference, Helsinki, in 2017 and support for department for conducting an educational meeting (PracticAllergy course)), ALK Abello (sponsorship to attend EAACI conference, Helsinki, in 2017), GSK, Mylan, Abbott, Allergy Therapeutics, Nutricia Danone and Biodiagnostics (support for conducting an educational meeting (PracticAllergy course)), outside the submitted work. A. Subramanian has nothing to disclose. N.J. Adderley has nothing to disclose. D.T. Zemedikun has nothing to disclose. G.V. Gkoutos has nothing to disclose. K. Nirantharakumar reports grants from NIHR, MRC, Diabetes UK and AstraZeneca, and personal fees from MSD, Sanofi and Boehringer Ingelheim, outside the submitted work.

Support statement: G.V. Gkoutos acknowledges support from H2020-EINFRA (731075) as well as support from the NIHR Birmingham ECMC, NIHR Birmingham SRMRC and NIHR Birmingham Biomedical Research Centre, and the MRC HDR UK (HDR UK/CFC/01).

\section{References}

1 Akdis M, Akdis CA. Mechanisms of allergen-specific immunotherapy: multiple suppressor factors at work in immune tolerance to allergens. J Allergy Clin Immunol 2014; 133: 621-631.

2 Buckley CD, McGettrick HM. Leukocyte trafficking between stromal compartments: lessons from rheumatoid arthritis. Nat Rev Rheumatol 2018; 14: 476-487. 
3 Robinson DS. T-cell cytokines: what we have learned from human studies. Paediatr Respir Rev 2004; 5: Suppl. A, S53-S58.

4 Romagnani S. Immunologic influences on allergy and the $\mathrm{T}_{\mathrm{H}} 1 / \mathrm{T}_{\mathrm{H}} 2$ balance. J Allergy Clin Immunol 2004; 113: 395-400.

5 Bach JF. The effect of infections on susceptibility to autoimmune and allergic diseases. N Engl J Med 2002; 347: 911-920.

6 Platts-Mills TA. The allergy epidemics: 1870-2010. J Allergy Clin Immunol 2015; 136: 3-13.

7 The International Study of Asthma and Allergies in Childhood (ISAAC) Steering Committee. Worldwide variation in prevalence of symptoms of asthma, allergic rhinoconjunctivitis, and atopic eczema. Lancet 1998; 351: $1225-1232$.

8 Garn H, Bahn S, Baune BT, et al. Current concepts in chronic inflammatory diseases: interactions between microbes, cellular metabolism, and inflammation. J Allergy Clin Immunol 2016; 138: 47-56.

9 Shen TC, Chen HJ, Wei CC, et al. Risk of asthma in patients with primary Sjögren's syndrome: a retrospective cohort study. BMC Pulm Med 2016; 16: 152.

10 Hsiao YP, Tsai JD, Muo CH, et al. Atopic diseases and systemic lupus erythematosus: an epidemiological study of the risks and correlations. Int J Environ Res Public Health 2014; 11: 8112-8122.

11 Hou YC, Hu HY, Liu IL, et al. The risk of autoimmune connective tissue diseases in patients with atopy: a nationwide population-based cohort study. Allergy Asthma Proc 2017; 38: 383-389.

12 Yeh JH, Kuo HT, Chen HJ, et al. Higher risk of myasthenia gravis in patients with thyroid and allergic diseases: a national population-based study. Medicine 2015; 94: e835.

13 Haahtela T, Holgate S, Pawankar R, et al. The biodiversity hypothesis and allergic disease: world allergy organization position statement. World Allergy Organ J 2013; 6: 3.

14 Cabieses B, Uphoff E, Pinart M, et al. A systematic review on the development of asthma and allergic diseases in relation to international immigration: the leading role of the environment confirmed. PLoS One 2014; 9: e105347.

15 Hussen HI, Moradi T, Persson M. The risk of type 1 diabetes among offspring of immigrant mothers in relation to the duration of residency in Sweden. Diabetes Care 2015; 38: 934-936.

16 Romagnani S. The increased prevalence of allergy and the hygiene hypothesis: missing immune deviation, reduced immune suppression, or both? Immunology 2004; 112: 352-363.

17 Booth N. What are the Read Codes? Health Libr Rev 1994; 11: 177-182.

18 Blak BT, Thompson M, Dattani H, et al. Generalisability of The Health Improvement Network (THIN) database: demographics, chronic disease prevalence and mortality rates. Inform Prim Care 2011; 19: 251-255.

19 Maguire A, Blak BT, Thompson M. The importance of defining periods of complete mortality reporting for research using automated data from primary care. Pharmacoepidemiol Drug Saf 2009; 18: 76-83.

20 Perkin $\mathrm{MR}$, Bader $\mathrm{T}$, Rudnicka AR, et al. Inter-relationship between rhinitis and conjunctivitis in allergic rhinoconjunctivitis and associated risk factors in rural UK children. PLoS One 2015; 10: e0143651.

21 Williams DC, Edney G, Maiden B, et al. Recognition of allergic conjunctivitis in patients with allergic rhinitis. World Allergy Organ J 2013; 6: 4.

22 Levesque LE, Hanley JA, Kezouh A, et al. Problem of immortal time bias in cohort studies: example using statins for preventing progression of diabetes. BMJ 2010; 340: b5087.

23 Al Sallakh MA, Vasileiou E, Rodgers SE, et al. Defining asthma and assessing asthma outcomes using electronic health record data: a systematic scoping review. Eur Respir J 2017; 49: 1700204.

24 Nissen F, Quint JK, Wilkinson S, et al. Validation of asthma recording in electronic health records: a systematic review. Clin Epidemiol 2017; 9: 643-656.

25 Dizon MP, Yu AM, Singh RK, et al. Systematic review of atopic dermatitis disease definition in studies using routinely collected health data. Br J Dermatol 2018; 178: 1280-1287.

26 Ban L, Langan SM, Abuabara K, et al. Incidence and sociodemographic characteristics of eczema diagnosis in children: a cohort study. J Allergy Clin Immunol 2018; 141: 1927-1929.

27 Adams J, Ryan V, White M. How accurate are Townsend Deprivation Scores as predictors of self-reported health? A comparison with individual level data. J Public Health 2005; 27: 101-106.

28 Zemedikun DT, Gray LJ, Khunti K, et al. Patterns of multimorbidity in middle-aged and older adults: an analysis of the UK Biobank data. Mayo Clin Proc 2018; 93: 857-866.

29 Fakih R, Diaz-Cruz C, Chua AS, et al. Food allergies are associated with increased disease activity in multiple sclerosis. J Neurol Neurosurg Psychiatry 2019; 90: 629-635.

30 Assa A, Frenkel-Nir Y, Tzur D, et al. Large population study shows that adolescents with celiac disease have an increased risk of multiple autoimmune and nonautoimmune comorbidities. Acta Paediatr 2017; 106: 967-972.

31 Kreiner E, Waage J, Standl M, et al. Shared genetic variants suggest common pathways in allergy and autoimmune diseases. J Allergy Clin Immunol 2017; 140: 771-781.

32 Jimenez-Morales S, Velazquez-Cruz R, Ramirez-Bello J, et al. Tumor necrosis factor-alpha is a common genetic risk factor for asthma, juvenile rheumatoid arthritis, and systemic lupus erythematosus in a Mexican pediatric population. Hum Immunol 2009; 70: 251-256.

33 Becker KG, Barnes KC. Underlying disease specificity of genetic loci in atopic dermatitis. J Invest Dermatol 2001; 117: $1325-1327$.

34 Demenais F, Margaritte-Jeannin P, Barnes KC, et al. Multiancestry association study identifies new asthma risk loci that colocalize with immune-cell enhancer marks. Nat Genet 2018; 50: 42-53.

35 Tang TS, Bieber T, Williams HC. Does "autoreactivity" play a role in atopic dermatitis? J Allergy Clin Immunol 2012; 129: 1209-1215.

36 Buehler U, Schulenburg K, Yurugi $\mathrm{H}$, et al. Targeting prohibitins at the cell surface prevents Th17-mediated autoimmunity. EMBO J 2018; 37: e99429.

37 Sorbello V, Ciprandi G, Di Stefano A, et al. Nasal IL-17F is related to bronchial IL-17F/neutrophilia and exacerbations in stable atopic severe asthma. Allergy 2015; 70: 236-240. 